\title{
FPGA IMPLEMENTATION OF ROAD NETWORK EXTRACTION USING MORPHOLOGICAL OPERATOR
}

\author{
CHINNATHEVAR SUJATHA ${ }^{\bowtie, 1}$ AND DHARMAR SELVATHI ${ }^{2}$ \\ ${ }^{1}$ ECE Department, SSM Institute of Engineering and Technology, Dindigul, Tamil Nadu, 624002, India; ${ }^{2}$ ECE \\ Department, Mepco Schlenk Engineering College, Sivakasi, Tamil Nadu, 626005, India \\ e-mail: sssujathac@yahoo.co.in,dselvathi@gmail.com \\ (Received: February 27, 2016: revised June 3, 2016; accepted June 10, 2016)
}

\begin{abstract}
In the remote sensing analysis, automatic extraction of road network from satellite or aerial images is the most needed approach for efficient road database creation, refinement, and updating. Mathematical morphology is a tool for extracting the features of an image that are useful in the representation and description of region shape. Morphological operator plays a significant role in the extraction of road network from satellite images. Most of the image processing algorithms need to handle large amounts of data, high repeatability, and general software is relatively slow to implement, so the system cannot achieve real-time requirements. In this paper, field programmable gate array (FPGA) architecture designed for automatic extraction of road centerline using morphological operator is proposed. Based on simulation and implementation, results are discussed in terms of register transfer level (RTL) design, FPGA editor and resource estimation. For synthesis and implementation of the above architecture, Spartan 3 XC3S400TQ144-4 device is used. The hardware implementation results are compared with software implementation results. The performance of proposed method is evaluated by comparing the results with ground truth road map as reference data and performance measures such as completeness, correctness and quality are calculated. In the software imple-mentation, the average value of completeness, correctness, and quality of various images are $90 \%, 96 \%$, and $87 \%$ respectively. In the hardware implementation, the average value of completeness, correctness, and quality of various images are $87 \%, 94 \%$, and $85 \%$ respectively. These measures prove that the proposed work yields road network very closer to reference road map.
\end{abstract}

Keywords: connected component, FPGA, morphological operation, performance measures, road network extraction, satellite image.

\section{INTRODUCTION}

Geographic Information Systems (GIS) needs an automatic road extraction process for updating their data (Hu et al., 2007). Manual updating of GIS database is costly, time consuming process and also there is a possibility of error in manual updating of the road network (Jin and Davis, 2005; Huang et al., 2012; Unsalan and Sirmacek, 2012). Therefore, automatic road feature extraction from high resolution satellite image is required to detect the road network in a robust manner. The objective of road feature extraction method is providing a binary mask in which true pixels represent road regions and false pixels indicate non-road regions. Many researchers are developing their own algorithm for automatic road detection and all the work has its own merits and demerits. Mathematical morphology is used to process and analyze the images and it plays a vital role in road network extraction. Some of the existing automatic road extraction works based on morpho-logical approach are discussed here.
Miao et al. (2014) proposed a method for road centerline extraction from classified image which consists of the three stages, extraction of feature points using tensor voting, kernel density estimation is used to find probability of each pixel being located on the road centerline and feature points are identified, centerline of road is formed by using geodesic method. The performance of this method is proved by comparing the quantitative measures with other methods. Shi et al. (2014) presented road extraction method for an urban area which includes two stages, image classified as road class and nonroad class and road class refined by shape features. The performance measures are evaluated and this is compared with other methods. Singh and Garg (2013) proposed a road network segmentation technique using adaptive global thresholding along with morphological operations. Road regions are segmented using average intensity values and morphological operators are used for further processing. They gave experimental result and evaluated their work by measuring quality para- 
meters. Automatic road extraction method using road intersection model is presented by Ahmed and Rahman (2011). The entire process of road intersection detection is divided into two sequential modules. The first module is the detection of road network using different morphological direction filter and the second module is an extraction of road intersection to determine road orientation. Correctness values are measured for evaluating this method.

The proposed work of this paper is road network boundary extraction using multi structural element (MSE) based morphological operator and centerline of the network is extracted using morphological operator. In this work, the road network extraction using the morphological operator with MSE and its hardware implementation is proposed. This proposed FPGA architecture is simulated using Verilog and synthesized on Spartan 3 FPGA device.

\section{MATERIAL AND METHODS}

Automatic road network extraction from high resolution satellite image includes the following stages (Sujatha and Selvathi, 2015). Approximated road region is segmented from satellite image using adaptive global thresholding method, morphological operators are applied to remove unwanted components in that output, extracting road pixels from segmented road region using connected component based trivial opening operations, edge detection using MSE morphological operator is applied to detect the boundary of the road network, and morphological thinning is applied to get centerline of the road network. The detailed methodology of proposed work is given in Fig. 1.

Adaptive global thresholding is applied to segment approximated road region from the satellite image. The histogram of the satellite image is analyzed and divided into four main sections to obtain the desired threshold value for segmentation (Singh and Garg, 2013). This approach is used in the case where single value thresholding will not function properly since the threshold value of pixel depends on its position within an image. Therefore, this technique is called as adaptive global thresholding. From this technique, approximated road regions are identified. The pixels lies in that region are assigned to the value 1 and remaining all the pixels are made to 0 . Now the gray image is converted into the binary image. For any pixel in an image, the set of pixels that are connected to that pixel is called connected component of an image (Dalla Mura et al., 2010). The analysis of connected components is mainly used for many automated image processing applications such as road map extraction, line detection, etc. Morphological operators are used to extracting the connected components of an image. Morphological dilation and erosion is the two most important basic morphological operator and other morphology operators are often derived from their combination. These morphological operators are used to extract the connected components of an image (Sujatha and Selvathi, 2015).

Dilation and erosion of a gray scale image $\mathrm{G}(\mathrm{x}, \mathrm{y})$ by a structural element $\mathrm{E}(\mathrm{s}, \mathrm{t})$ are denoted as in Eq. 1 and Eq. 2 respectively.

$$
\begin{aligned}
& \mathrm{G} \oplus \mathrm{E}(\mathrm{x}, \mathrm{y})=\max \{\mathrm{G}(\mathrm{x}-\mathrm{s}, \mathrm{y}-\mathrm{t})+\mathrm{E}(\mathrm{s}, \mathrm{t})\}, \\
& \mathrm{G} \odot \mathrm{E}(\mathrm{x}, \mathrm{y})=\min \{\mathrm{G}(\mathrm{x}-\mathrm{s}, \mathrm{y}-\mathrm{t})-\mathrm{E}(\mathrm{s}, \mathrm{t})\} .
\end{aligned}
$$

The morphological opening of $\mathrm{G}$ and $\mathrm{E}$ is defined as erosion followed by dilation and Morphological closing is defined as the dilation followed by erosion. Opening and closing are given in Eq. 3 and Eq. 4 respectively.

$$
\begin{aligned}
& G^{\circ} E=(G \odot E) \oplus E, \\
& G \cdot E=(G \oplus E) \oplus E .
\end{aligned}
$$

Let $\mathrm{G}$ is an image and connected components in an image are denoted as $G(1), G(2), G(3), \ldots$. The first non-zero pixel in an image is $\mathrm{p}$ and assigned that value as $\mathrm{X} 0$. The connected pixels with this $\mathrm{X}_{0}$ pixel are extracted as per the Eq. 5. That $X_{0}$ is dilated with structural element $\mathrm{E}$ and dilated output is intersected with an image that output is $X_{1}$. If $X_{1} \neq X_{0}$ then this iteration process is repeated, if $X_{1}=X_{0}$ then iteration is stopped and $X_{1}$ is the first set of the connected component. The above process is continued until all the non-zero pixels are grouped in any one of the connected components.

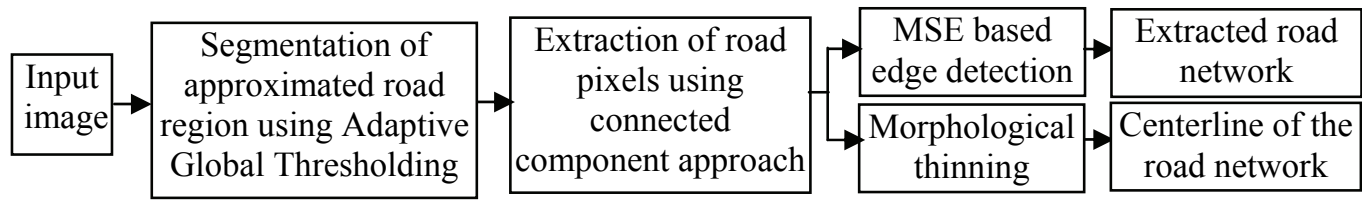

Fig. 1. Methodology of the proposed work. 


$$
\mathrm{X}_{\mathrm{k}}=\left(\mathrm{X}_{\mathrm{k}-1} \oplus \mathrm{E}\right) \cap \mathrm{G} \mathrm{k}=1,2,3, \ldots
$$

Some of these connected components are nonroad regions and these unwanted components are removed by using the Trivial opening method. Trivial opening is defined by Serra and Vincent (1992) and it is used to extract the connected components based on some criteria. Let $\mathrm{G}$ be an image, $\{\mathrm{G}(\mathrm{n}) \mid \mathrm{n}=1,2,3$, $\ldots, N\}$ is a sequence of connected components in the image $\mathrm{G}$ which are extracted using Eq. 5. The trivial opening is defined with a condition $\mathrm{T}$, as follows in Eq. 6.

$$
\begin{array}{r}
\tau_{0}=\{G(i), \text { if } G(i) \text { satisfy the condition } T, \\
\phi, \text { otherwise }\}
\end{array}
$$

Therefore, $\tau_{\mathrm{o}}$ is the trivial opening associated with condition $\mathrm{T}$. The trivial opening is used to extract the required portion of an image based on condition $T$. This operation removes the connected component which is not satisfied the condition $\mathrm{T}$. If connected component satisfied the condition $\mathrm{T}$, then entire region of that components are preserved. So shape and size are not disturbed by this opening. Road areas are easily filtered by trivial opening because roads have appeared as an identical region and long features in satellite images. The roads are extracted by selecting the condition as the long axes of a minimum ellipse for trivial opening operation. The Trivial opening for road detection is expressed as follows in Eq. 7.

$$
\begin{array}{r}
\text { Ro }=\{G \mid \text { Long axis of axis of minimum ellipse } \\
\text { enclosing } G(\mathrm{i})>=\mathrm{T}\},
\end{array}
$$

where $\mathrm{G}$ is an image and $\mathrm{G}(\mathrm{i})$ is connected components of an image. Thus, resultant image Ro has the connected components which are greater than $\mathrm{T}$ and this resultant image consists of road regions and all unwanted regions are removed.

\section{ROAD BOUNDARY EXTRACTION USING MSE BASED MORPHOLOGICAL EDGE DETECTION}

The resultant image of the trivial opening method consists of road network regions. The boundary of this network is extracted using MSE based morphological edge detection. Edges of an image are detected using morphological gradient edge detector and it is denoted by $E_{g}(G)$. The edge is detected by using the Eq. 8.

$$
\mathrm{E}_{\mathrm{g}}(\mathrm{G})=(\mathrm{G} \circ \mathrm{E}) \oplus \mathrm{E}-(\mathrm{G} \cdot \mathrm{E}) \oplus \mathrm{E},
$$

where $\mathrm{G}$ is an image and $\mathrm{E}$ is a structural element.
The performance of the morphological based edge detection mainly depends on the choice of structural element (SE). The shape and size of SE affect the edge detection result. Single SE is only sensitive to image edge which has the specific direction (Dalla Mura et al., 2010). Thus, the MSE based edge detection algorithm is developed, that is detecting the image edges with different directions, and each SE detects an image edge in the corresponding direction. The larger SE detects the thicker edge than smaller SE based edge detection method. Thus, the size of SE is $3^{*} 3$ and the direction angles of SE are $0^{\circ}, 45^{\circ}, 90^{\circ}$ and $135^{\circ}$ are chosen. These SEs are shown in Fig. 2.

\begin{tabular}{cccc}
\hline $\begin{array}{c}\text { SE1 with } \\
0^{\circ} \text { angle }\end{array}$ & $\begin{array}{c}\text { SE2 with } \\
45^{\circ} \text { angle }\end{array}$ & $\begin{array}{c}\text { SE3 with } \\
90^{\circ} \text { angle }\end{array}$ & $\begin{array}{c}\text { SE4 with } \\
135^{\circ} \text { angle }\end{array}$ \\
\hline$\left[\begin{array}{lll}0 & 0 & 0 \\
1 & 1 & 1 \\
0 & 0 & 0\end{array}\right]$ & {$\left[\begin{array}{lll}0 & 0 & 1 \\
0 & 1 & 0 \\
1 & 0 & 0\end{array}\right]$} & {$\left[\begin{array}{lll}0 & 1 & 0 \\
0 & 1 & 0 \\
0 & 1 & 0\end{array}\right]$} & {$\left[\begin{array}{lll}1 & 0 & 0 \\
0 & 1 & 0 \\
0 & 0 & 1\end{array}\right]$} \\
\hline
\end{tabular}

Fig. 2. Multistructural elements used in this method

These structure elements comprise almost all the directions of lines extend in the image. By using these four SEs, horizontal, vertical and diagonal edges are extracted. Morphological gradient edge detection in $0^{\circ}$ is detected using the Eq. 9.

$$
\mathrm{E}_{1}(\mathrm{G})=(\mathrm{G} \circ \mathrm{SE} 1) \oplus \mathrm{SE} 1-(\mathrm{G} \cdot \mathrm{SE} 1) \Theta \mathrm{SE} 1 .
$$

Morphological gradient edge detection in $45^{\circ}$ is detected using the Eq. 10.

$$
\mathrm{E}_{2}(\mathrm{G})=(\mathrm{G} \circ \mathrm{SE} 2) \oplus \mathrm{SE} 2-(\mathrm{G} \cdot \mathrm{SE} 2) \odot \mathrm{SE} 2 .
$$

Morphological gradient edge detection in $90^{\circ}$ is detected using the Eq. 11.

$$
\mathrm{E}_{3}(\mathrm{G})=(\mathrm{G} \circ \mathrm{SE} 3) \oplus \mathrm{SE} 3-(\mathrm{G} \cdot \mathrm{SE} 3) \Theta \mathrm{SE} 3 .
$$

Morphological gradient edge detection in $135^{\circ}$ is detected using the Eq. 12.

$$
\mathrm{E}_{4}(\mathrm{G})=(\mathrm{G} \circ \mathrm{SE} 4) \oplus \mathrm{SE} 4-(\mathrm{G} \cdot \mathrm{SE} 4) \odot \mathrm{SE} 4,
$$

where $\mathrm{G}$ is a gray scale image, SE1, SE2, SE3, SE4 are structural elements and values are given in Fig. 2. Image fusion method is used to get the final edge map of an image as given Eq. 13.

$$
\mathrm{E}_{\mathrm{m}}(\mathrm{G})=\sum \mathrm{E}_{\mathrm{i}}(\mathrm{G}) / \mathrm{M}
$$

where $i=1,2, \ldots, M . E_{m}(G)$ is the final detected edge of original image $\mathrm{M}$ is the number of SE. The obtained edge map is in gradient format that is converted into binary format. The MSE based edge detection is slightly modified to get the road boundaries and block diagram of that method is shown in Fig. 3.This method needs dilation, erosion, summation and intersection blocks. 


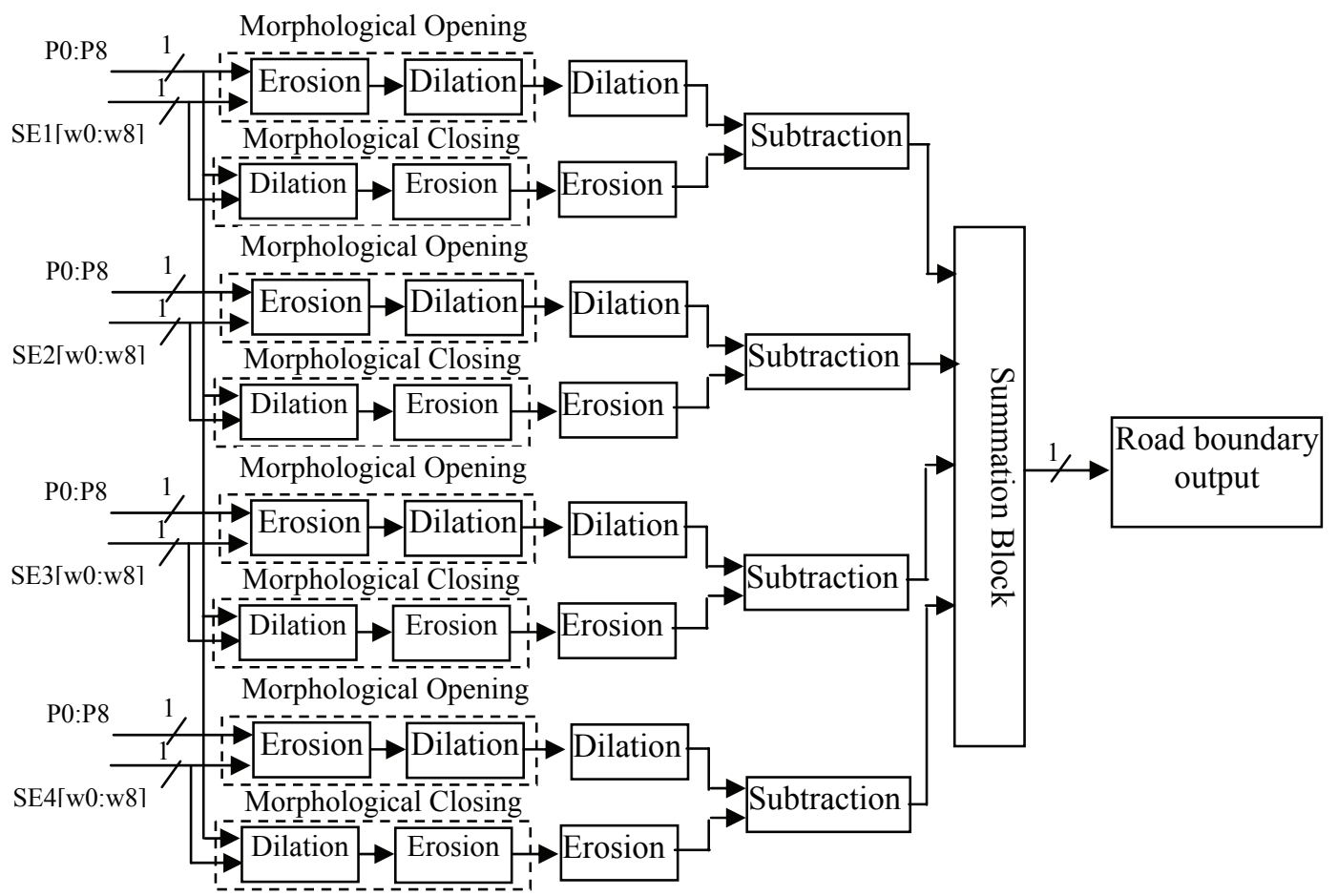

Fig. 3. Block diagram for boundary of road network

\section{EXTRACTION OF CENTERLINE OF ROAD NETWORK}

The resultant image of trivial opening have the regions of the entire road network and by using MSE based edge detection method boundary of the road network is obtained. The morphological thinning operation is applied to get the centerline of the road network. The thinning of an image $\mathrm{G}$ by a structural element $\mathrm{E}$ is defined as in Eq. 14.

$$
\mathrm{G} \otimes \mathrm{E}=\mathrm{G}-\left(\mathrm{G}^{*} \mathrm{E}\right)=\mathrm{G} \cap\left(\mathrm{G}^{*} \mathrm{E}\right)^{\mathrm{C}},
$$

where $\left(\mathrm{G}^{*} \mathrm{E}\right)$ represent the hit-or-miss transform used to identify specified configuration of pixels (Reddy et $a l ., 2012)$ and it is given in Eq. 15.

$$
\mathrm{G}^{*} \mathrm{E}=\left(\mathrm{G} \odot \mathrm{E}_{1}\right) \cap\left(\mathrm{G}^{\mathrm{C}} \odot \mathrm{E}_{2}\right),
$$

where E1, E2 are subsets of E related with object and

\begin{tabular}{|c|c|c|c|c|}
\hline \begin{tabular}{|l|} 
Input \\
Image
\end{tabular} & $\rightarrow \begin{array}{c}\text { Matlab Program } \\
\text { (Image to hexa file } \\
\text { converter })\end{array}$ & \begin{tabular}{|c|}
$\begin{array}{c}\text { FPGA Architecture } \\
\text { for road network } \\
\text { extraction }\end{array}$ \\
\end{tabular} & \begin{tabular}{|c} 
Matlab Program \\
(Hexa file to image \\
converter $)$
\end{tabular} & $\begin{array}{c}\text { Extracted } \\
\text { road network } \\
\text { output }\end{array}$ \\
\hline
\end{tabular}
background respectively.

Fig. 4. Flow Diagram for FPGA implementation of road network extraction.

\section{FPGA IMPLEMENTATION OF ROAD NETWORK EXTRACTION}

The FPGA architecture is proposed for road centerline extraction using morphological operator. For real time application, hardware implementation is most needed. The overall flow diagram for FPGA implementation of road network extraction is shown in Fig. 4. The Matlab program is used to convert image data into hexa data file. FPGA architecture designed to process these data and produces the output which is converted into image format by using Matlab program.

\section{FPGA ARCHITECTURE FOR ROAD EXTRACTION}

The block diagram of proposed FPGA architecture is shown in Fig. 5. It consists of adaptive threshold module, connected component labelling, trivial opening, Morphological edge detection, and morphological thinning. 


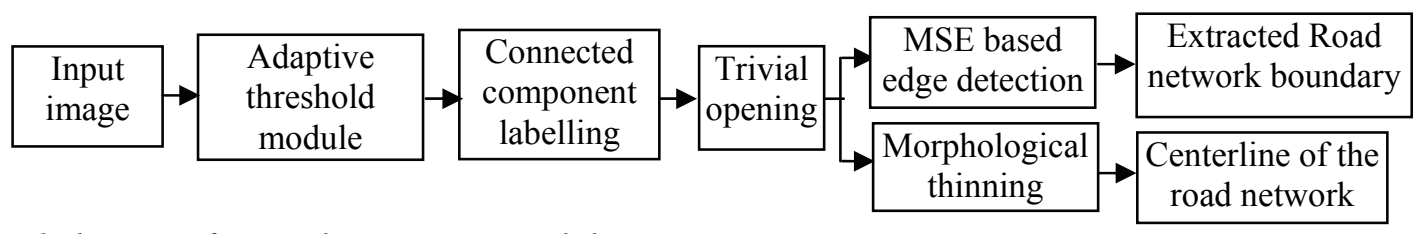

Fig. 5. Block diagram for road extraction module.

The input image is converted into binary using adaptive threshold method. The architecture is shown in Fig. 6. In this architecture, $\mathrm{P}$ is the 8-bit pixel value of an image and $\mathrm{T}$ is the 8-bit threshold value which is found using adaptive threshold method. If pixel value $\mathrm{P}$ is greater than threshold value $\mathrm{T}$, then the output is 1 or output is 0 . Thus, 8 -bit input pixel is converted into binary format. This threshold output contains some unwanted region. The unwanted region is removed using connected component extraction. Connected components are extracted using the iterative Eq. 7. The algorithm behind the extraction of the connected component is as follows. Converted binary image from the adaptive threshold method is assigned as matrix A, any one of SE from Fig. 2. is chosen and assigned as $\mathrm{B}$. The first non-zero element position in the input matrix $\mathrm{A}$ is found. The matrix $\mathrm{X}$ is initialized with zeros and place 1 in the non-zero element position found in the previous step. Morphological dilation using the structural element $\mathrm{B}$ on matrix $\mathrm{X}$ is performed. The dilated matrix is intersected with the matrix A. Check whether $Y==X$. If both are not equal, then perform dilation and intersection again. If both are same, then iteration is stopped. The non-zero elements position in the $\mathrm{Y}$ is labelled with number $\mathrm{L}$. where $\mathrm{L}$ is the number of connected component in that image A. Similarly, place zero in those positions in the input matrix A. The block diagram for connected component labelling is shown in Fig. 7.

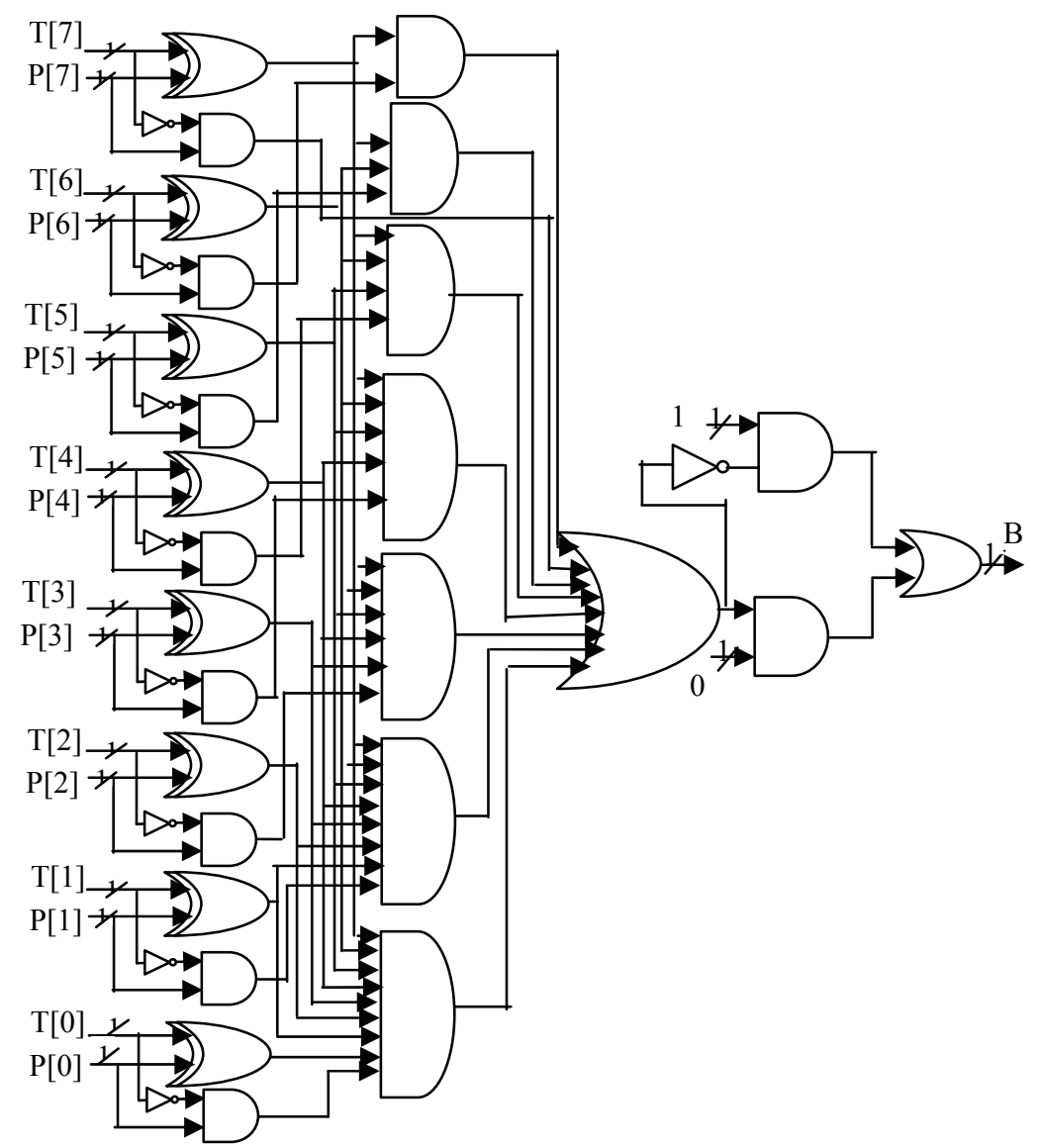

Fig. 6. Block diagram for adaptive threshold module. 


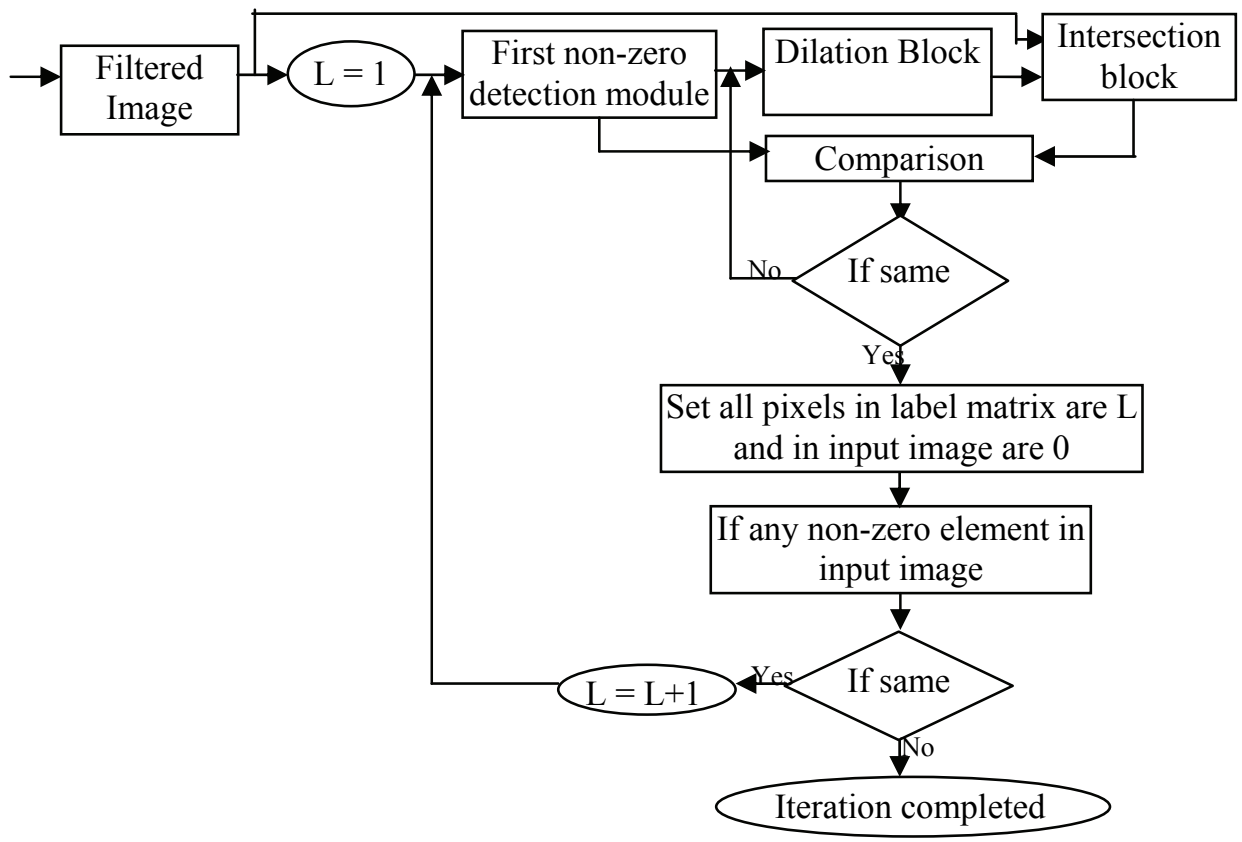

Fig. 7. Block diagram for connected component labelling.

The connected component block diagram consists of nonzero detection, dilation, and intersection modules. The architecture of these modules is given in Fig. 8. The first non-zero detection block is used to find the first non-zero values in the given matrix and it has nine input and nine output. Table 1 shows the conditions to find the first zero among nine neighborhood pixels. The internal architecture for this block is shown in Fig. 8.

The first non-zero block is followed by dilation block which is used to find the maximum among the neighbors. That morphological dilation architecture and an internal circuit of the maximum block (MAXB) used in dilation is also given in Fig. 9. The dilated output is followed by intersection block. Two binary number is intersected by using the AND operations. The comparison block is used to compare the output of intersection block with non-zero block output. If both outputs are same, iteration is stopped otherwise the entire process is repeated as shown in the Fig. 7. By using these processes connected component is identified and the unwanted component is removed by using the trivial opening method. In the trivial opening method, the length of each connected component is measured and long length component is retained remaining are removed. These processes give the road region in an image. Road boundary is extracted from the processed road region image using MSE based morphological edge detection method. The architecture of road boundary detection using MSE based morphological operator is shown in Fig. 9.

Table 1. Conditions to find first nonzero pixels.

\begin{tabular}{|c|c|c|c|c|c|c|c|c|c|c|c|c|c|c|c|c|c|c|}
\hline \multicolumn{1}{|c|}{ Input A } & \multicolumn{1}{|c|}{ Output A } & Output \\
\hline P0 & $\mathrm{P} 1$ & $\mathrm{P} 2$ & $\mathrm{P} 3$ & $\mathrm{P} 4$ & $\mathrm{P} 5$ & $\mathrm{P} 6$ & $\mathrm{P} 7$ & $\mathrm{P} 8$ & $\mathrm{~N} 0$ & $\mathrm{~N} 1$ & $\mathrm{~N} 2$ & $\mathrm{~N} 3$ & $\mathrm{~N} 4$ & $\mathrm{~N} 5$ & $\mathrm{~N} 6$ & $\mathrm{~N} 7$ & $\mathrm{~N} 8$ & Expression \\
\hline 1 & $\mathrm{x}$ & $\mathrm{x}$ & $\mathrm{x}$ & $\mathrm{x}$ & $\mathrm{x}$ & $\mathrm{x}$ & $\mathrm{x}$ & $\mathrm{x}$ & 1 & 0 & 0 & 0 & 0 & 0 & 0 & 0 & 0 & N0 = P0 \\
\hline 0 & 1 & $\mathrm{x}$ & $\mathrm{x}$ & $\mathrm{x}$ & $\mathrm{x}$ & $\mathrm{x}$ & $\mathrm{x}$ & $\mathrm{x}$ & 0 & 1 & 0 & 0 & 0 & 0 & 0 & 0 & 0 & N1 = P1'.P0 \\
\hline 0 & 0 & 1 & $\mathrm{x}$ & $\mathrm{x}$ & $\mathrm{x}$ & $\mathrm{x}$ & $\mathrm{x}$ & $\mathrm{x}$ & 0 & 0 & 1 & 0 & 0 & 0 & 0 & 0 & 0 & N2 = P2'P1.P0 \\
\hline 0 & 0 & 0 & 1 & $\mathrm{x}$ & $\mathrm{x}$ & $\mathrm{x}$ & $\mathrm{x}$ & $\mathrm{x}$ & 0 & 0 & 0 & 1 & 0 & 0 & 0 & 0 & 0 & N3 = P3'P2.P1.P0 \\
\hline 0 & 0 & 0 & 0 & 1 & $\mathrm{x}$ & $\mathrm{x}$ & $\mathrm{x}$ & $\mathrm{x}$ & 0 & 0 & 0 & 0 & 1 & 0 & 0 & 0 & 0 & N4 = P4'P3.P2.P1.P0 \\
\hline 0 & 0 & 0 & 0 & 0 & 1 & $\mathrm{x}$ & $\mathrm{x}$ & $\mathrm{x}$ & 0 & 0 & 0 & 0 & 0 & 1 & 0 & 0 & 0 & N5 = P5'P4.P3.P2.P1.P0 \\
\hline 0 & 0 & 0 & 0 & 0 & 0 & 1 & $\mathrm{x}$ & $\mathrm{x}$ & 0 & 0 & 0 & 0 & 0 & 0 & 1 & 0 & 0 & N6 = P6'P5.P4.P3.P2.P1.P0 \\
\hline 0 & 0 & 0 & 0 & 0 & 0 & 0 & 1 & 0 & 0 & 0 & 0 & 0 & 0 & 0 & 0 & 1 & 0 & N7 = P7'.P6.P5.P4.P3.P2.P1.P0 \\
\hline 0 & 0 & 0 & 0 & 0 & 0 & 0 & 0 & 1 & 0 & 0 & 0 & 0 & 0 & 0 & 0 & 0 & 1 & N8 = P8'.P7.P6.P5.P4.P3.P2.P1.P0 \\
\hline
\end{tabular}




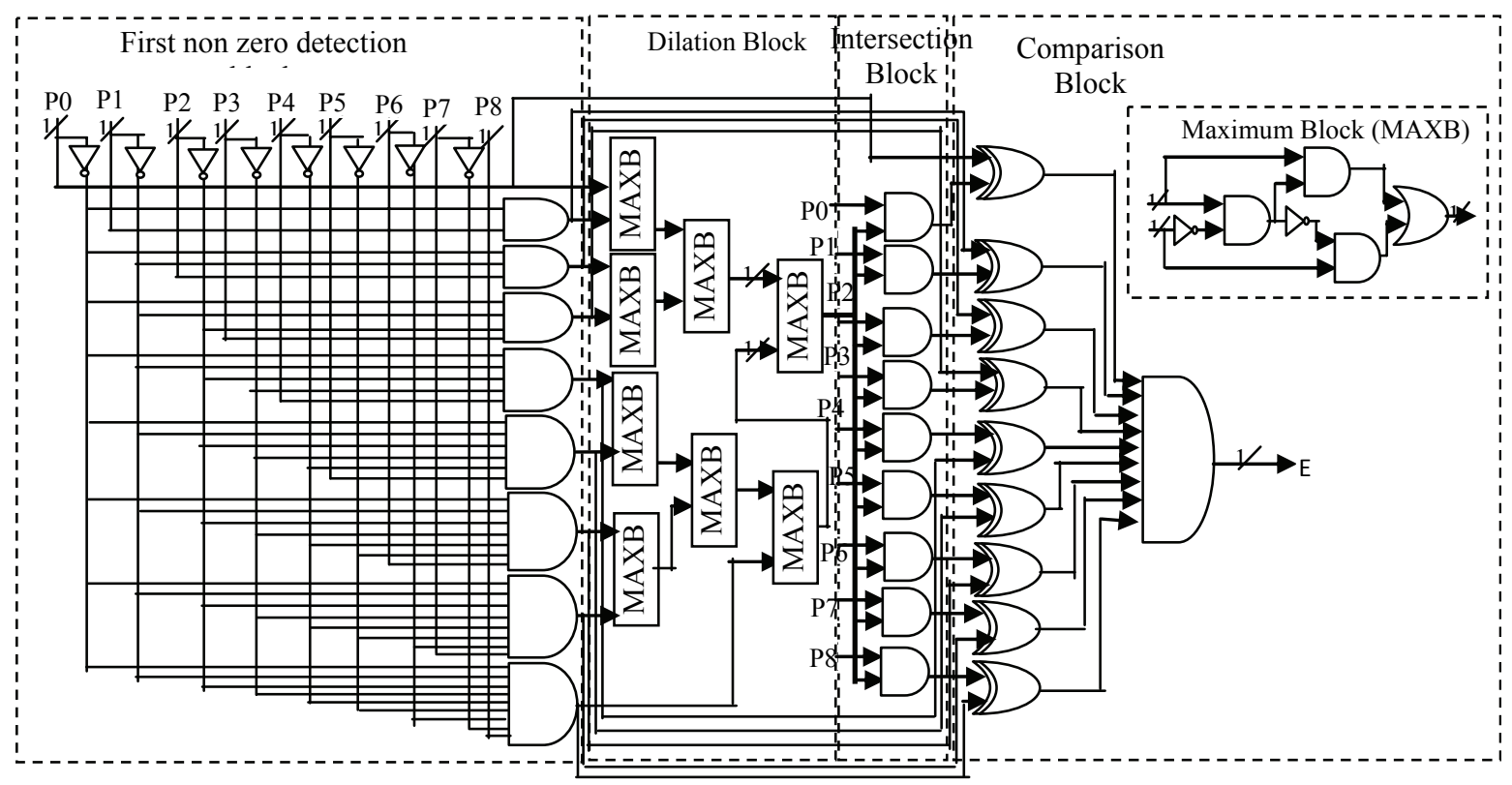

Fig. 8. International architecture for connected component labelling.

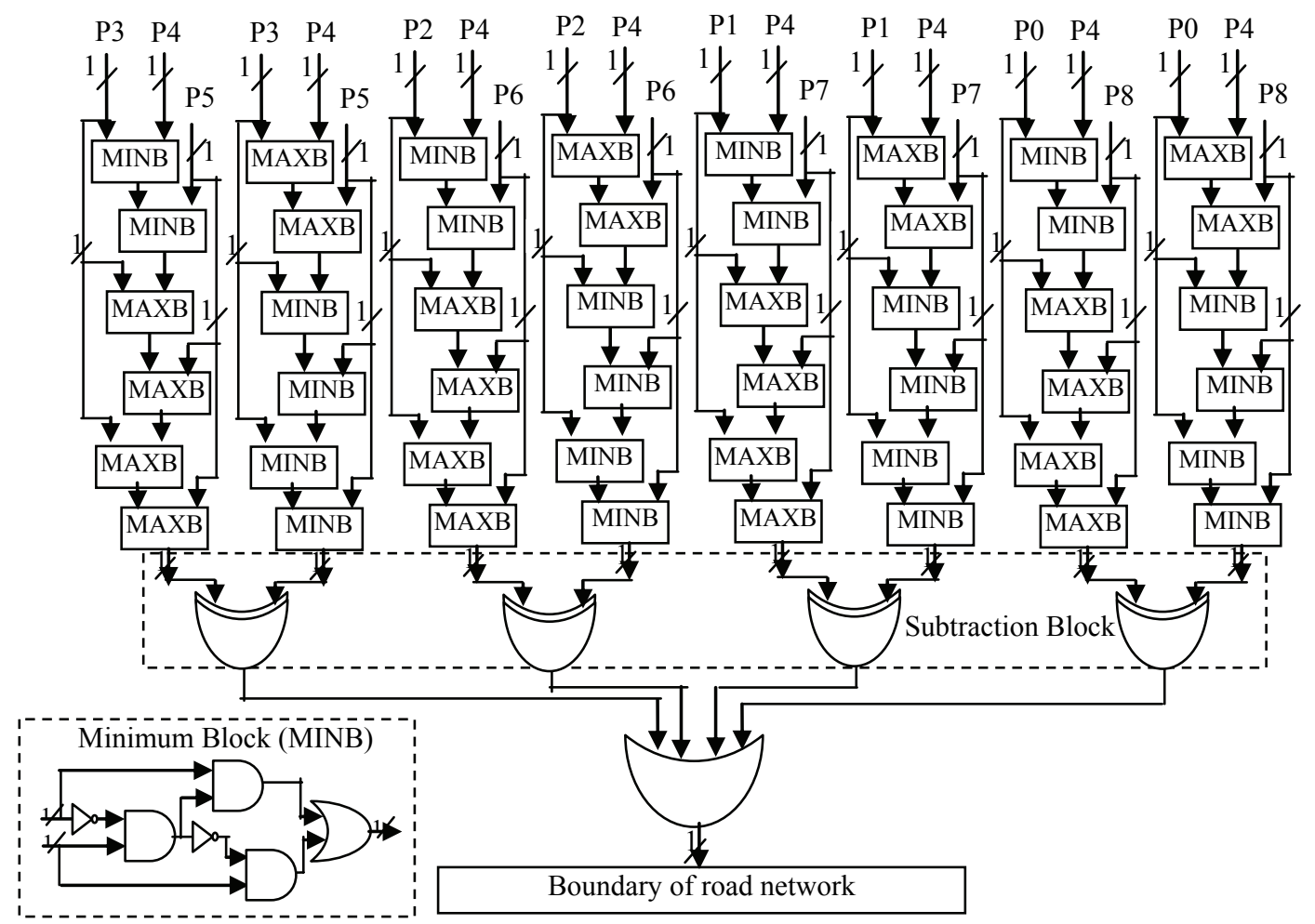

Fig. 9. Architecture diagram of morphological based road boundary detector.

The architecture consists of the maximum block (MAXB), minimum block (MINB), subtraction block and finally adds the four outputs to get the road pixels. The architecture provides the boundary of the road network. The centerline of that network is extracted using morphological thinning operations. The thinning of an image $\mathrm{G}$ by SE is defined in terms of the hit-ormiss transform. The block diagram for morphological thinning is shown in Fig. 10 and internal architecture for thinning is shown in Fig. 11.

The eroded original image is intersected with eroded complemented pixel and intersection block is subtracted with the original image to get road centerline. Thus by using the proposed FPGA architecture road boundary and the centerline of the road network are produced. 


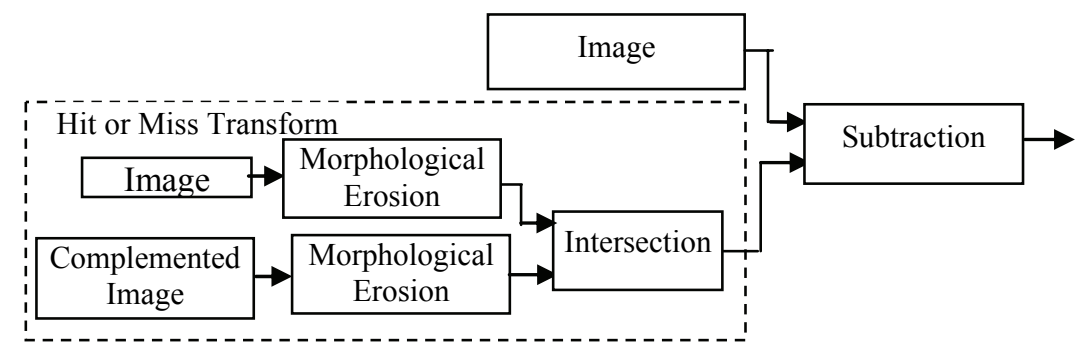

Fig. 10. Block diagram of morphological thinning.

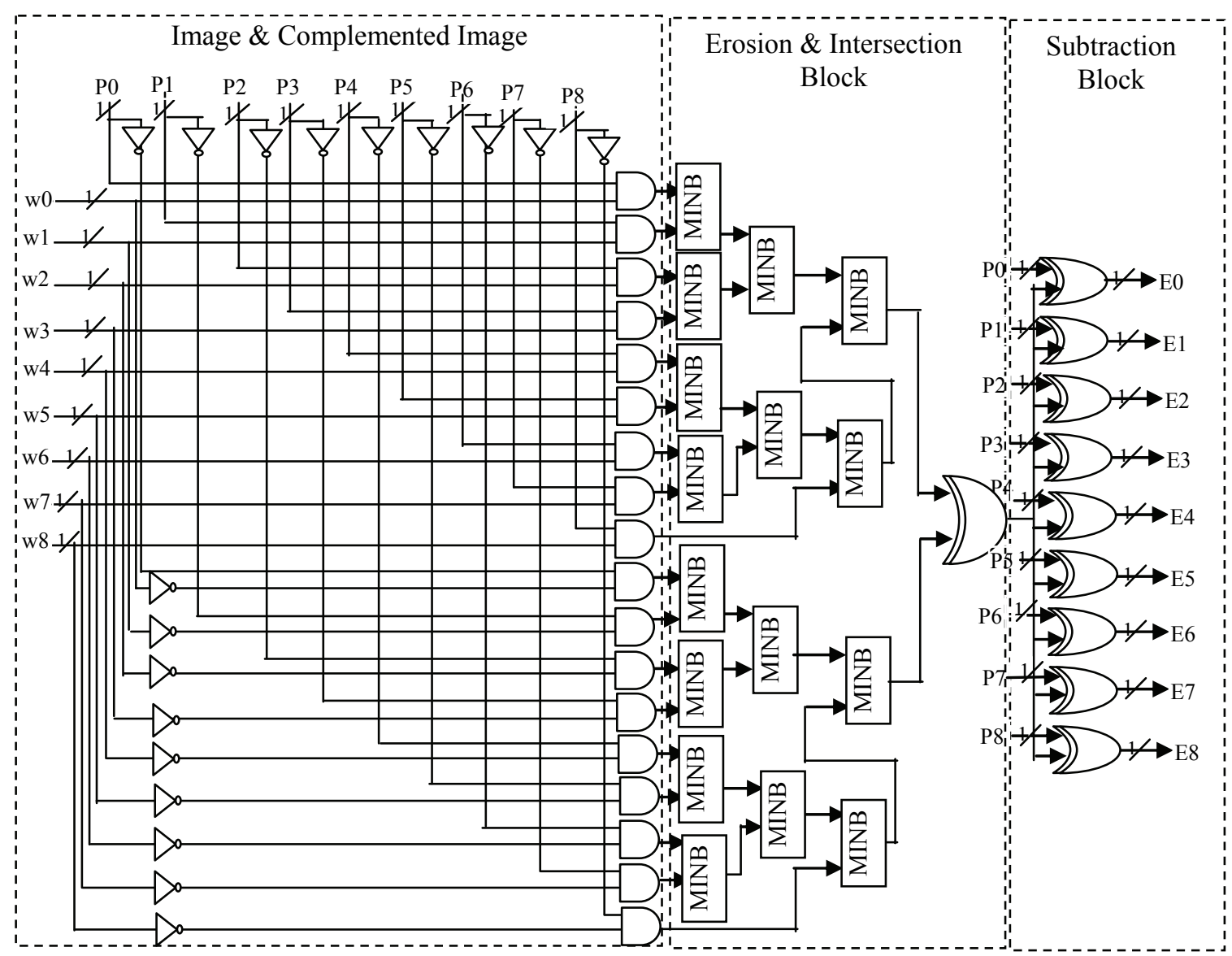

Fig. 11. Internal architecture of morphological thinning.

\section{RESULTS}

The proposed architecture is implemented on a Xilinx Spartan 3 FPGA device. The sub-modules of the system are individually coded in Verilog and simulated using Xilinx ISE simulator.

The synthesis and implementation in device Spar$\tan 3$ XC3S400TQ144 help to determine the resource utilization which indicates the amount of resources exploited by the entire architecture. Table 2 shows the number of Flip-Flops, LUT, Slice, I/Os utilized and timing summary.

The Table 2 proves that proposed system requires very less area only $14 \%$ of flip-flops and $12 \%$ LUT is used. Thus proposed system provides better performance with less device utilization. It indicates that the minimum period required for this implementation is $8.835 \mathrm{~ns}$ and the maximum frequency is $113.186 \mathrm{MHz}$. 
Table 2. FPGA resource utilization and timing summary of proposed architecture.

\begin{tabular}{lc}
\hline \multicolumn{1}{c}{ Resources } & Count \\
\hline Number of Slices & $810 / 3584(22 \%)$ \\
Number of Slice Flip-Flops & $1012 / 7168(14 \%)$ \\
Number of 4 input LUTs & $897 / 7168(12 \%)$ \\
Number of IOBs & $22 / 97(20 \%)$ \\
Number of GCLKs & $1 / 8(12 \%)$ \\
Minimum period & $8.835 \mathrm{~ns}$ \\
Maximum Frequency & $113.186 \mathrm{MHz}$ \\
Minimum input arrival time & $10.364 \mathrm{~ns}$ \\
before clock & \\
Maximum output required time & $7.430 \mathrm{~ns}$ \\
after clock & 195912 kilobytes \\
Total memory usages
\end{tabular}

The designed FPGA architectures for road boundary network extraction and road centerline extraction are tested on satellite images and obtained hardware results are compared with software implementation results which are given in Fig. 12.

This comparison proves that hardware implementation result is merely equal to software result.

\section{PERFORMANCE EVALUATION OF ROAD EXTRACTION}

The performance of the road network detection is also analyzed quantitatively using various measures. Performance measures such as completeness, correctness, and quality are the most important parameters for the evaluation of the road extraction. To measure these parameters, extracted road network is compared with manually drawn ground truth road map (Heipke et al., 1997). Digitized manually drawn road map is obtained using the software GIMP (GNU Image Manipulation Program). GIMP is high quality freely available software for image processing applications (Solomon, 2009). The matched extracted road data are calculated as True Positive (TP) and the unmatched extracted data is calculated as False Positive (FP).The unmatched reference data are calculated as False Negative (FN). The completeness is the ratio between matched reference road data with the total length of reference road map. The completeness is calculated by using the Eq. 16 .

$$
\text { Comp } \%=\mathrm{TP} /(\mathrm{TP}+\mathrm{FN}) * 100 \text {. }
$$

The correctness represents the percentage of correctly extracted road data and it is measured by using the following Eq. 17.

$$
\text { Corr } \%=\mathrm{TP} /(\mathrm{TP}+\mathrm{FP}) * 100 .
$$

The goodness of the road extraction result is measured using the quality measures. The value of quality is calculated by using the following Eq. 18 .

Qual $\%=\mathrm{TP} /(\mathrm{TP}+\mathrm{FP}+\mathrm{FN}) * 100$

The performance measures such as Completeness, Correctness, and Quality are evaluated for proposed work on various test images. These measures are calculated for hardware implementation results and tabulated in Table 3.

This comparison Table proves that hardware implementation result is almost equal to software result.

Table 3. Performance measures comparison of hardware and software implementation result of the proposed method.

\begin{tabular}{c|c|c|c|c}
\hline Images & Algorithm & Completeness \% & Correctness \% & Quality \% \\
\hline \multirow{2}{*}{ Test Image 1 } & Software implementation & 89.30 & 99.17 & 88.64 \\
& Hardware implementation & 88.02 & 97.73 & 88.18 \\
\hline \multirow{2}{*}{ Test Image 2 } & Software implementation & 87.12 & 94.16 & 82.65 \\
& Hardware implementation & 85.28 & 92.10 & 81.91 \\
\hline \multirow{2}{*}{ Test Image 3 } & Software implementation & 88.74 & 95.37 & 85.07 \\
& Hardware implementation & 84.51 & 91.19 & 83.10 \\
\hline \multirow{2}{*}{ Test Image 4 } & Software implementation & 94.76 & 98.21 & 93.15 \\
& Hardware implementation & 92.71 & 96.15 & 91.39 \\
\hline \multirow{2}{*}{ Test Image 5 } & Software implementation & 90.16 & 94.64 & 85.78 \\
& Hardware implementation & 88.47 & 93.85 & 82.57 \\
\hline \multirow{2}{*}{ Average } & Software implementation & 90.02 & 96.31 & 87.06 \\
& Hardware implementation & 87.80 & 94.20 & 85.43 \\
\hline
\end{tabular}



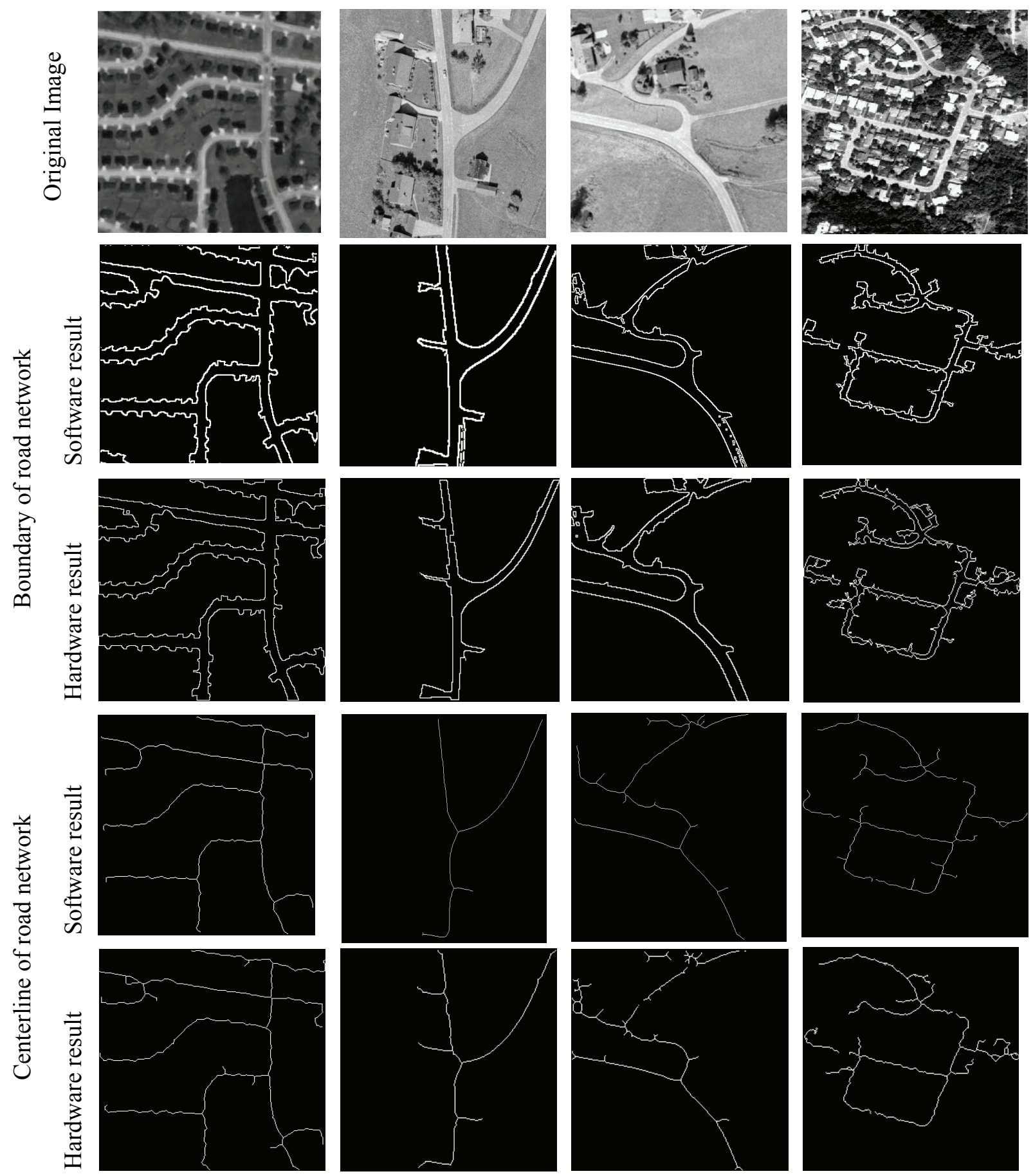

Fig. 12. Comparison of hardware implementation results with software implementation.

\section{DISCUSSION}

Road centerline extraction of high resolution satellite images using morphological operation is proposed in this work. The proposed work includes the following steps such as segmentation of approximated road regions using adaptive global threshold method; trivial opening with the criteria of a long axis of the mini- mum ellipse of the connected component is applied to extract road pixels from the segmented region. MSE based morphological edge detection is applied to detect the boundary of the road network. Thinning is applied to detect the centerline of the road network. FPGA architecture is proposed for this road network extraction. The designed architecture is simulated using Verilog, synthesized using Xilinx ISE. This architecture is implemented on Spartan 3 XC3S400-4TQ144 
device. The device utilization is very less and time delay is $8.835 \mathrm{~ns}$ ( $3.678 \mathrm{~ns}$ logic, $5.157 \mathrm{~ns}$ route). The total memory required for implementation of the developed method has been found as 195912 kilobytes. This architecture uses only $14 \%$ slice FFs on Xilinx Spartan 3 XC3S400-4TQ144. The proposed method is implemented on various satellite images and results in both software and hardware implementation are given. The completeness, correctness, and quality measures are evaluated for both hardware and software implementation and results are compared. These comparisons proved that hardware result is close to software result.

\section{REFERENCES}

Ahmed B, Rahman MF (2011). Automatic road extractions from high resolution satellite imagery using road intersection model in urban areas. Comput Eng Intell Syst 2:82-93.

Dalla Mura M, Benediktsson JA, Waske B, Bruzzone L (2010). Morphological attribute profiles for the analysis of very high resolution images. IEEE T Geosci Remote 48:3747-62.

Heipke C, Mayer H, Wiedemann C, Jamet O (1997). Evaluation of automatic road extraction. Int Arch Photogram Remote Sens 32:151-60.

Hu J, Razdan A, Femiani JC, Cui M, Wonka P (2007). Road network extraction and intersection detection from aerial images by tracking road footprints. IEEE $\mathrm{T}$ Geosci Remote 45:4144-57.

Huang Z, Zhang J, Wang L, Xu F (2012). A feature fusion method for road line extraction from remote sensing image. Proc IEEE International Symposium on Geo- science and Remote Sensing, 2012 July 22;52-5.

Jin X, Davis CH (2005). An integrated system for automatic road mapping from high-resolution multi-spectral satellite imagery by information fusion. Inform Fusion 6:257-73.

Miao Z, Wang B, Shi W, Wu H (2014). A method for accurate road centerline extraction from a classified image. IEEE J Appl Earth Obs Remote Sens 7:4762-71.

Reddy GVR, Vijaya Kumar V, Reddy MA (2012). Morphological texture synthesis algorithm using pixel and patch based approach. Int J Sci Eng Res 3:1-4.

Serra J, Vincent L (1992) An overview of morphological filtering. Circ Syst Signal Proc 11:47-108.

Shi W, Miao Z, Wang Q, Zhang H (2014). Spectral-spatial classification and shape features for urban road centerline extraction. IEEE Geosci Remote Lett 11:788-92.

Singh PP, Garg RD (2013). Automatic road extraction from high resolution satellite image using adaptive global thresholding and morphological operations. J Indian Soc Remote Sens 41:631-40.

Solomon RW (2009). Free and open source software for the manipulation of digital images. Am J Roentgenol 192:330-4.

Sujatha C, Selvathi D (2015). Connected component-based technique for automatic extraction of road centerline in high resolution satellite images. EURASIP J Image Video Proc 8:1-16.

Unsalan C, Sirmacek B (2012). Road network detection using probabilistic and graph theoretical methods. IEEE T Geosci Remote 50:4441-53. 